\title{
ON NORMAL LATTICES AND WALLMAN SPACES
}

GEORGE M. EID

\author{
Department of Mathematics \\ John Jay College of Criminal Justice \\ The City University of New York \\ 445 West 59 th Street \\ New York, NY 10019, U.S.A.
}

(Received March 28, 1988 and in revised form June 20, 1988)

\begin{abstract}
Let $X$ be an abstract set and $\Omega$ a lattice of subsets of $X$. The notion of $\Omega$ being mildly normal or slightly normal is investigated. Also, the general Wallman space with an alternate topology is investigated, and for $\Omega$ not necessarily disjunctive, an analogue of the Wallman space is constructed.
\end{abstract}

KEY WORDS AND PHRASES. Normal lattice, 0-1 valued measures, Wallman space, disjunctive lattice, almost compact, almost countably compact. 1980 AMS SUBJECT CLASSIFICATION CODES. 28A60, 28 A52.

\section{INTRODUCTION.}

In the first part of this paper, we consider lattices which satisfy conditions weaker than normality; more precisely mildly normal and slightly normal lattices. We give examples of such lattices, and then investigate the preservation of these properties under lattice extension and restriction.

Next, we investigate spaces which are related to the general Wallman space. First, instead of considering the customary topology on the Wallman space, we introduce another topology and show how topological properties reflect strongly to the underlying lattice. Then we consider the case of a lattice which is not necessarily disjunctive and construct an associated Wallman type space. This work generalizes that of Liu (see Section 5).

We adhere to standard lattice terminology that can be found, for example, in [1], [2], [3], [4], [5]. However, in section 2, we summarize the principal lattice concepts and notations that w111 be used throughout the paper for the convenience of the reader. We then precede to the consideration of mildly normal and slightly normal lattices in section 3, and then to analogues of the general Wallman space in sections 4 and 5. We finally note that most of the results hold equally well for abstract lattices.

2. DEFINITIONS AND NOTATIONS.

a) Let $X$ be an abstract set and $\Omega$ a lattice of subsets of $X$. We shall always assume, without loss of generality for our purposes, that $\phi, X \varepsilon \Omega$. The set whose general element $L^{\prime}$ is the complement of an element $L$ of 2 is ri. red by '. $^{\prime}$ 'is 
said to be complement generated iff, for every $L$ of $\Omega$ there exists a sequence

$\left\{L_{n}\right\}_{n=1}^{\infty}$ in $\Omega$ such that $L=n_{n=1}^{\infty} L_{n}^{\prime}$. $\Omega$ is separating if. for any two elements $\mathrm{x} \neq \mathrm{v}$ of $\mathrm{x}$, there exists an element $\mathrm{L} \varepsilon \Omega$ such that $\mathrm{x} \in \mathrm{L}$ and $\mathrm{y} \in \mathrm{L}, \quad \Omega$ is $T_{2}$ if, for any two elements $x \neq y$ of $x$, there exists $A, B \in$ such that $x \in A^{\prime}, y \in B^{\prime}$ and $A^{\prime} \cap B^{\prime}=\phi_{0} \quad \Omega$ is said to be disjunctive iff, for every $x \in X$ and $L \varepsilon \Omega$, if $x \notin L$ then there exists an $\hat{L} \varepsilon \Omega$, such that $x \in \hat{L}$ and $L \cap \hat{L}=\phi$. $\Omega$ is regular iff, for every $x \in X$ and every $L \varepsilon \Omega$, if $x \notin L$ then there exists $L_{1}, L_{2} \varepsilon \Omega$ such that $x \in L_{1}^{\prime}, L_{C} \subset L_{2}^{\prime}$ and $L_{1}^{\prime} \cap L_{2}^{\prime}=\phi$. $\quad \Omega$ is normal iff, for any $L_{1}, L_{2} \varepsilon \Omega$, if $\mathrm{L}_{1} \cap \mathrm{L}_{2}=\phi$ then there exists $\hat{\mathrm{L}}_{1}, \hat{\mathrm{L}}_{2} \varepsilon \Omega$ such that $\mathrm{L}_{1} \subset \hat{\mathrm{L}}_{1}^{\prime}, \mathrm{L}_{2} \subset \hat{\mathrm{L}}_{2}^{\prime}$ and $\hat{\mathrm{L}}_{1}^{\prime} \cap \hat{\mathrm{L}}_{2}^{\prime}=\phi \cdot \Omega 1 \mathrm{~s}$ lindelof iff, for every $L_{\alpha} \varepsilon \Omega$; $a \in A$, if $\prod_{\alpha} L_{\alpha}=\phi$ then for a countable subcollection $\left\{\mathrm{L}_{\alpha_{1}}\right\}$ of $\left\{\mathrm{L}_{\alpha}\right\} ; \quad \bigcap_{1=1}^{\infty} \mathrm{L}_{\alpha_{1}}=\phi$. $\quad \Omega$ is compact $1 \mathrm{ff}$, for every $\mathrm{L}_{\alpha} \varepsilon \Omega, \quad \alpha \varepsilon A$, if $\bigcap_{\alpha} L_{\alpha}=\phi$ then for some finite subcollection $\left\{L_{\alpha}\right\}$ of $\left\{L_{\alpha}\right\} ; \bigcap_{i=1}^{n} L_{\alpha}=\phi$. Next, consider any two lattices $\Omega_{1}, \Omega_{2}$ of subsets of $X_{0} \Omega_{1}$ is said to semi-separate $\Omega_{2}$ or for abbreviation $\left(\Omega_{1}\right.$ s.s $\left.\Omega_{2}\right)$ iff, for every $L_{1} \varepsilon \Omega_{1}$ and every $L_{2} \varepsilon \Omega_{2}$ if $L_{1} \cap L_{2}=0$ then there exists $\hat{\mathrm{L}}_{1} \varepsilon \Omega_{1}$ such that $\mathrm{L}_{2} \subset \hat{\mathrm{L}}_{1}$ and $\mathrm{L}_{1} \cap \hat{\mathrm{L}}_{1}=\phi$. $\Omega_{1}$ is said to separate $\Omega_{2}$ if for any $\mathrm{L}_{2}, \hat{\mathrm{L}}_{2} \varepsilon \Omega$, if $\mathrm{L}_{2} \cap \hat{\mathrm{L}}_{2}=\phi$ there exist $\mathrm{L}_{1}, \hat{\mathrm{L}}_{1} \in \Omega_{1}$ such that $\mathrm{L}_{2} \subset \mathrm{L}_{1}, \hat{\mathrm{L}}_{2} \subset \hat{\mathrm{L}}_{1}$ and $L_{1} \cap \hat{L}_{1}=\phi$. We denote by $\tau \Omega+1$, set whose general element is the intersecti,n if arbitrary subsets of $\Omega$. b) Let $A$ h. $\rightarrow, y$ algebra of subsets of $X$. A measure on $A$ is defined to be a function, $\mu$ from $A \leftrightarrow R$ such that $\mu$ is bounded and finitely additive. The algebra of subsets of $X$ generated by $\Omega$ is denoted by $A(\Omega)$. If $x \in X$, then $\mu_{x}$ is the measure concentrated at $x$ so $\mu_{i}(i)=\left\{\begin{array}{lll}1 & \text { if } & x \in A \\ 0 & \text { if } & x \notin A\end{array}\right.$ where $A \notin A(j)$. The set whose general element is a measure on $A(\Omega)$ is denoted $M(\Omega)$. Note that, since every element of $M(\Omega)$ is equal to the difference of nonnegative elements of $M(\Omega)$, , without loss of generality, we may work excl. i ivoly with nonnegative elements of $M(\Omega)$. Let $\mu \varepsilon M(\Omega), \mu$ is $\Omega$ regular if for any $A \in A(\Omega) ; \mu(A)=\sup \{\mu(L) ; L \subset A, L \varepsilon \Omega\}$. The set whose general element is an element of $M(\Omega)$ which is $\delta$ regular is denoted by $M_{R}(\Omega)$. An element $\mu \in M(\Omega)$ is $\sigma$ smooth on $\Omega$, if $L_{n} \varepsilon \Omega, n=1,2, \ldots$ and $L_{n}+\phi$ then $\mu\left(L_{n}\right) \rightarrow 0$. The set whose general element is an element of $M(\Omega)$ whish is $\sigma$ smooth on $\Omega$ is denoted by $M_{\sigma}(\Omega)$. We say that $\mu$ is orsmooth on $A(\Omega)$ if $A_{n} \varepsilon A(\Omega), n=1,2 \ldots$ and $A_{n}+\phi t h \rho_{i}{ }_{1}\left(A_{n}\right) \rightarrow 0$. The set whose general element is an element of $M(\Omega)$ which osmooth on $A(\Omega)$ is denoted by $M^{\sigma}(\Omega)$. Note that if $\mu \in M_{R}(\Omega)$, then $\mu \in M_{R}^{\sigma}(\Omega)$ iff $\mu \in M_{\sigma}(\Omega) . I(\Omega), I_{R}(\Omega), I_{\sigma}(\Omega), I^{\sigma}(\Omega), I_{R}^{\sigma}(\Omega)$ are the subsets of the corresponding $M^{\prime} s$ consisting of the non-trivial zero-one valued measures. For $\mu \varepsilon M(\Omega)$, the support of $\mu, S(\mu)=\cap\{L \varepsilon \Omega, \mu(L)=\mu(X)\}$. L is replete iff, whenever $\mu \varepsilon I_{R}^{\sigma}(\Omega)$ then $S(\mu) \neq \phi$. A premeasure on $\Omega$ is defined to be a function $\pi$ from $\Omega$ to $\{0,1\}$ such that $\pi(\phi)=0, \pi(A)<\pi(B)$ for every $A \subset B$ where $A, B \in \Omega$ and if $\pi(A)=\pi(B)=1$ then $\pi(A \cap B)=1$. $\Pi(\Omega)$ denotes the set of all premeasures on $\Omega_{0}$ c) As an immediate consequence of Zorn's Lemma, we have, for every $\mu \varepsilon I(\Omega)$, there exists an element $\nu \varepsilon I_{R}(\Omega)$ such that $\mu<\nu$ on $\Omega$ or simply $(\mu<v(\Omega))$. Also, for any two lattices $\Omega_{1}, \Omega_{2}$ of subsets of $X$, if $\Omega_{1} \subset \Omega_{2}$, then for every $\mu \varepsilon I_{R}\left(\Omega_{1}\right)$, there exists a $\nu \varepsilon I_{R}\left(\Omega_{2}\right)$ such that $\left.\nu\right|_{A(\Omega)}=\mu$ and such that a $\nu$ is unique if $\Omega_{1}$ separates $\Omega_{2}$. Moreover, $\Omega$ is normal iff, for $\mu \in I(\Omega), \mu<v_{1}(\Omega), \mu<v_{2}(\Omega)$ where $v_{1}, v_{2} \varepsilon I_{R}(\Omega)$ then 
$\nu_{1}=\nu_{2}$. $\Omega$ is regular iff, for any $\mu_{1}, \mu_{2} \varepsilon I(\Omega) ; \mu_{1}<\mu_{2}(\Omega)$ then $S\left(\mu_{1}\right)=S\left(\mu_{2}\right)$. The result $\mu_{x} \varepsilon I_{R}(\Omega)$ iff $\Omega$ is disjunctive leads us to the Wallman Topology which is obtained by taking the totality of all $W(L)=\left\{\mu \varepsilon I_{R}(\Omega) ; \mu(L)=1\right.$ for $\left.L \varepsilon \Omega\right\}$ as a base for the closed sets on $I_{R}(\Omega)$. For a disjunctive $\Omega, I_{R}(\Omega)$ with the tW( $(\Omega)$ of closed sets 18 a compact $T_{1}$ space and will be $T_{2}$ iff $\Omega$ is normal and is called the general Wallman space associated with $X$ and $\Omega$. Also, for a disjunctive $\Omega$ and $A, B \varepsilon A(\Omega)$, $W(A)$ is a lattice with respect to union and Intersection. Moreover, $W\left(A^{\prime}\right)=(W(A))^{\prime}$, $W(A(\Omega))=A(W(\Omega)), W(A)=W(B)$ iff $A=B$ and $W(A) \subset W(B)$ iff $A \subset B$. Now, we note that, if $\Omega$ is disjunctive so is $W(\Omega)$, and in addition to each $\mu \varepsilon M(\Omega)$, there exists a $\hat{\mu} \varepsilon M(W(\Omega))$ defined by $\mu(A)=\hat{\mu}(W(A))$ for all $A \in A(\Omega)$ such that the map $\mu \rightarrow \hat{\mu}$ is one-to-one and onto; moreover $\mu \in M_{R}(\Omega)$ iff $\hat{\mu}_{\varepsilon} M_{R}(W(\Omega))$.

\section{ON NORMAL LATTICES.}

In this section, we elaborate on the notion of a normal lattice, investigate lattices which satisfy weaker conditions, and discuss their interrelations under the extension and restriction properties. Throughout this section $\Omega_{1} \Omega_{1}$, and $\Omega_{2}$ will denote lattices of subsets of the set $X$. Note that, for $L_{1} \varepsilon \Omega ; 1=1,2,3$; $\Omega$ is normal iff $L_{1} \subset L_{2}^{\prime} \cup L_{3}^{\prime}$, then $L_{1}=A_{1} \cup B_{1} ; A_{1} \subset L_{2}^{\prime}, B_{1} \subset L_{3}^{\prime} ; A_{1}, B_{1} \varepsilon \Omega$

THEOREM 3.1. Let $\Omega$ be normal, $\mu \in I(\Omega)$ and $G=\left\{L^{\prime} \varepsilon \Omega^{\prime} ; \hat{L} \subset L^{\prime}, \mu(\hat{L})=1, \hat{L}_{\varepsilon \Omega}\right\}$ then, $G$ is a prime $\Omega^{\prime}-f i 1 t e r$.

PROOF. Clearly, $G$ is an $\Omega^{\prime}-$ filter since $\phi G, \mu\left(\hat{L}_{1} \cap \hat{L}_{2}\right)=1$ and if $L_{1} \varepsilon G ; L_{1} \subset L_{2}^{\prime}$ then $L_{2}^{\prime} \varepsilon G$. In addition, if $L_{1}^{\prime} \cup L_{2}^{\prime} \varepsilon G$, then $\hat{L} \subset L_{1} \cup L_{2}^{\prime}$ and by the normality of $\Omega$, $L=\hat{L}_{1} \cup \hat{L}_{2}$ with $1=\mu(\hat{L})<\mu\left(\hat{L}_{1}\right)+\mu\left(\hat{\mathrm{L}}_{2}\right)$ then elther $\mu\left(\hat{\mathrm{L}}_{1}\right)=1$ or $\mu\left(\hat{\mathrm{L}}_{2}\right)=1$ and so either $L_{1}^{\prime} \varepsilon G$ or $L_{2}^{\prime} \varepsilon G$. Thus, $G$ is a prime $\Omega^{\prime}$ - filter.

THEOREM 3.2. Suppose $\Omega_{1} \subset \Omega_{2}$ and $\Omega_{1}$ separates $\Omega_{2}$. Then, $\Omega_{1}$ is normal iff $\Omega_{2}$ is normal.

PROOF. (1) Suppose $\Omega_{1}$ is normal then by the separation. It is clear that $\Omega_{2}$ is normal. (11) Suppose $\Omega_{2} 18$ normal. Let $\mu \in I\left(\Omega_{1}\right), \mu<v_{1}, v_{2}\left(\Omega_{1}\right) ; v_{1}, v_{2} \varepsilon I_{R}\left(\Omega_{1}\right)$. Extend $v_{1}, v_{2}$, to $\lambda_{1}, \lambda_{2} \varepsilon I_{R}\left(\Omega_{2}\right)$ and $\mu$ to $\tau \in I\left(\Omega_{2}\right)$. Suppose there exists an $L_{2} \varepsilon \Omega_{2} ; \tau\left(\Omega_{2}\right)=1$, but $\lambda_{1}\left(L_{2}\right)=0$, then $\lambda_{1}\left(L_{2}^{\prime}\right)=1$ and so there exists $\hat{L}_{2} \varepsilon \Omega_{2} ; \hat{L}_{2} \subset L_{2}^{\prime}$ $\lambda_{1}\left(\hat{\mathrm{L}}_{2}\right)=1$. By the separation, there exists $\hat{\mathrm{L}}_{1}, \mathrm{~L}_{1} \varepsilon \Omega, \hat{\mathrm{L}}_{1} \supset \hat{\mathrm{L}}_{2}, \mathrm{~L}_{1} \supset \mathrm{L}_{2}$. Consequent $1 \mathrm{y}$, $\lambda_{1}\left(\hat{L}_{1}\right)=1$ but $\tau\left(L_{1}\right)=1$ then $\lambda_{1}\left(L_{1}\right)=1$ wh1ch 18 a contradiction. Therefore, $\tau<\lambda_{1}\left(\Omega_{2}\right)$ and simflarly $\tau<\lambda_{2}\left(\Omega_{2}\right)$. Thus, by the normality of $\Omega_{2}, \lambda_{1}=\lambda_{2}$ then $v_{1}=v_{2}$ and $80 \Omega_{1} 18$ normal.

DEFINITION 3.1. $\Omega$ is sald to be mildiy normal, if for all $\mu \varepsilon I_{\sigma}(\Omega)$, there exists a unique $\nu \in I_{R}(\Omega) ; \mu<V(\Omega)$.

DEFINITION 3.2. $\Omega$ is sald to be almost countably compact, if for all

$\mu \varepsilon I_{R}\left(\Omega^{\prime}\right), \mu \varepsilon I_{\sigma}(\Omega)$.

DEFINITION 3.3. $\Omega$ 1s sald to be prime replete, if for all $\mu \varepsilon I_{\sigma}(\Omega), S(\mu) \neq \phi$. THEOREM 3.3. If $\Omega$ is regular and prime replete, then $\Omega$ is mildly normal.

PROOF. If $\Omega$ is not mildly normal, then there exists $\mu \varepsilon I_{\sigma}(\Omega), \nu_{1} \neq v_{2} \varepsilon I_{R}(\Omega)$ and $\mu<v_{1}(\Omega), \mu<v_{2}(\Omega)$. Then, there exists $L_{1}, L_{2} \varepsilon \Omega ; L_{1} \cap L_{2}=\phi \cdot v_{1}\left(L_{1}\right)=v_{2}\left(L_{2}\right)=1$ and $\nu_{1}\left(L_{2}\right)=\nu_{2}\left(L_{1}\right)=0$. But since $\Omega 18$ regular, $S(\mu)=S\left(\nu_{1}\right) \subset L_{1}$ and $S(\mu)=S\left(\nu_{2}\right) \subset L_{2}$. Then, $S(\mu) \subset\left(L_{1} \cap L_{2}\right)=\phi$ and so $S(\mu)=\phi$ which is a contradiction since $\Omega$ is prime replete. Thus, $\Omega$ must be mildiy normal. 
THEOKEM 3.4. If 18 is regular and lindelot, then is is mild ly normal.

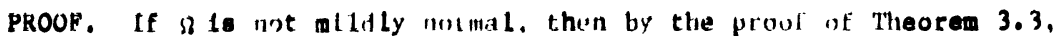
$\left.S(\mu) C: L_{1} \cap L_{2}\right)=\phi$. Moruover, $p=r_{u} L_{u}=\vdots(u)=S\left(v_{1}\right)=: s\left(v_{2}\right)$ w1th ${ }_{11}\left(L_{\alpha}\right)=1$ and

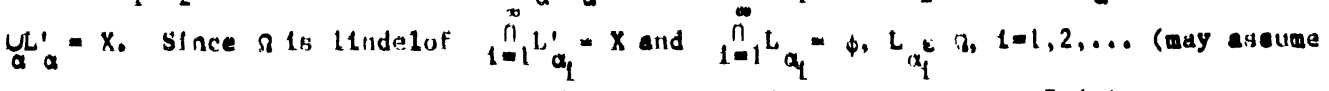
$\left.L_{\alpha_{1}}{ }\right)$, but $\mu\left(\alpha_{1}\right)=1$ for all 1 which is a contradiction since $\mu \in I_{\sigma}(\Omega)$. Thus, $\Omega$ muat be mildy normal.

THEOREM 3.5. If a ts almost countably compact and int ldiy normal, then $\Omega$ lo normal. PROOF. Suppose $\Omega$ is almost contitably compact, and $\mu \& I(\Omega)$. Then $\mu<v \in I_{R}\left(\Omega^{\prime}\right)$ on $\Omega^{\prime}$, and $80 \nu<\mu(\Omega)$; $\nu \in I_{g}(\Omega)$. If $\zeta<t_{R}(\Omega)$ and $\mu: \delta(\Omega)$, then $v<\delta(\Omega)$, $\nu$ \& $I_{g}(\Omega)$. Since $\Omega$ ls mildiy uormal the rest of the proot 19 obvious. THEOREM 3. 6 . Suppose $\Omega_{1} \subset \Omega_{2}$ and $i_{1}$ separates $\left.\Omega_{2}\right)^{\circ}$ 'Then, $\pi_{2}$ to mildly normal te $\Omega_{1} 19$ mildly normal.

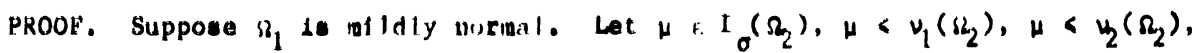
$\nu_{1}, \nu_{2} \in I_{R}\left(\Omega_{2}\right)$, then $\mu<v_{1} \mid\left(\Omega_{1}\right)$, ; $: \nu_{2}\left|\left(\Omega_{1}\right) ; \nu_{1}\right|, \nu_{2} \mid \cdot I_{R}\left(\Omega_{1}\right)$ and $\mu E I_{\sigma}\left(\Omega_{2}\right)$, hence $\nu_{1}\left|=v_{2}\right|$ since $\Omega_{1}$ is mildly normal. Thus $\nu_{1}=v_{2}$ slice $\Omega_{1}$ separates $\Omega_{2}$ and so $\Omega_{2} 18$ mildy nornat.

DEFINITIUN 3.4. $\Omega_{2}$ 1s said to be $\Omega_{1}$-countably bounded, if given $B_{n} E B_{2}, B_{n}+\phi$, there exiots an $A_{1} \& \delta_{1}: A_{n}+\phi$ and $B_{n} \in A_{n}$.

In the next theorem, we will see when a partial converse of the theorem 3.6 is true.

THEOREM 3.7. Suppose $z_{1}$ separates $s_{2}$, and $s_{2}$ is $s t_{1}$-countably bounded, then $1 f$ $\Omega_{2}$ is intidly normal so is $\Omega_{1}$.

PRoOF. Suppose $\Omega_{2} 18 \mathrm{mildly}$ normul and $\Omega_{1}$ separates $\Omega_{2}$. Let $\mu \in I_{\sigma}\left(\Omega_{1}\right)$ and $\mu<\nu_{1}\left(\Omega_{1}\right), \mu<v_{2}\left(\Omega_{1}\right)$ where $\nu_{1}, \nu_{2} t t_{k}\left(\Omega_{1}\right)$. Extend $\mu$ to $t \in I\left(\Omega_{2}\right)$. By separation, $v_{1}$ and $v_{2}$ extend $u$ iquely tu $\zeta_{1}$ and $\zeta_{2}$ reepectively, where $\zeta_{1}, \zeta_{2} \in I_{R}\left(\Omega_{2}\right)$, and $t$ - $r_{1}, \zeta_{2}\left(L_{2}\right)$. Let $B_{n}+\phi, B_{n}$. $\Omega_{2}$. Stuce $\Omega_{2}$ to $\pi_{1}$-countably bounded, there exists $A_{n}+\phi, B_{n} \subset A_{n}, A_{n} \varepsilon \Omega_{1}$. Then $t\left(B_{n}\right)<\tau\left(A_{n}\right)-\mu\left(A_{n}\right)=0$ for some $n$. Then $T \in I_{0}\left(\Omega_{2}\right)$. Slince $\Omega_{2}$ is mildiy normal, $\zeta_{1}=r_{2}$ and hence $v_{1}=v_{2}$ o $\Omega_{1}$ is otldiy normal.

DEFINITION 3.5. $\Omega$ L salil tu be countably paracompact if, whenever $A_{n}+\phi, A_{n} \in \Omega$, there existe $B_{n} \in S_{i} A_{n} \subset B_{n}^{\prime}$ and $B_{n}^{\prime}+\phi$

DEFINLTION 3.6. $\Omega$ is sald to be alightly normal, if for all $\mu \in I_{\sigma}\left(\Omega^{\prime}\right)$, there extote a unlque $\nu \in I_{R}(\Omega): \mu<\mathcal{V}(\Omega)$

THEOREM 3.8. If is to regular and lindelof, then $\Omega$ io slightiy normal.

PROOF. Suppose $\Omega 18$ not silghtly unrmal. Let $\mu$ r. $I_{\sigma}\left(\Omega^{\prime}\right), \mu<\nu_{1}(\Omega), \mu<y_{2}(\Omega)$

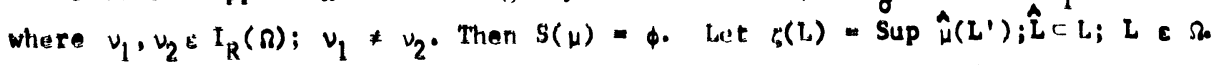
Then, $\zeta$ is a premeasure, t.e. $r, e \pi(\Omega)$. Mureover, $S(\mu)=S(\zeta)$ elnce $\Omega$ la regular. Also $\zeta \in \pi_{\sigma}(\Omega)$, and eince $\Omega$ is lindelof, $S\left(\zeta_{\zeta}\right) \neq \phi$ then $S(\mu) \neq \phi$ which is a contradiction. Thus, si must be slightly normal.

THEOREM 3.9. Suppose $1{ }_{1}$ epparates $52_{2}$. If $13_{1}$ to slighty nurmal, then $\pi_{2}$ is lightly normal.

PKOOF. Let $\mu \in I_{j}\left(\Omega_{2}^{\prime}\right) ; \mu<v_{1}\left(\Omega_{2}\right), \mu \leqslant \nu_{2}\left(\Omega_{2}\right)$ where $\nu_{1}, \nu_{2}<1_{R}\left(\Omega_{2}\right)$, than by separation $\mu \mid\left\langle\nu_{1}\left(s \Omega_{1}\right), \mu\right|\left\langle\nu_{2}\left|\left(\Omega_{1}\right) ; \mu\right|=I_{0}\left(s_{1}^{\prime}\right)\right.$ and $\nu_{1}\left|, \nu_{2}\right|=I_{R}\left(\Omega_{1}\right)$. Therefore, 
$\nu_{1}\left|=\nu_{2}\right|$ since $\Omega$ is slightly normal and so $\nu_{1}=\nu_{2}$ by separation and hence $\Omega_{2}$ is slightly normal.

LFMMA 3.1. If $\Omega$ is complement generated, then $\Omega$ is slightly normal.

PROOF. If $\Omega$ is complement generated, then $I_{\sigma}\left(\Omega^{\prime}\right) \subset I_{R}(\Omega)$, that is, if $\mu \varepsilon I_{\sigma}\left(\Omega^{\prime}\right)$ there exists a unique $\nu \in I_{R}(\Omega) ; \mu<\chi(\Omega)$. Thus, $\Omega$ is slightly normal.

In the next theorem, we will see when a partial converse of theorem 3.9 is true. THEOREM 3.10. Suppose $\Omega_{1}$ separates $\Omega_{2^{*}}$ If $\Omega_{2}$ is mildly normal and countably paracompact then $\Omega_{1}$ is slightly normal.

PROOF. Let $\mu \varepsilon I_{\sigma}\left(\Omega_{1}^{\prime}\right) ; \mu<\nu_{1}\left(\Omega_{1}\right), \mu<\nu_{2}\left(\Omega_{1}\right) ; \nu_{1}, \nu_{2} \varepsilon I_{R}\left(\Omega_{1}\right)$. Extend $\mu$ to $\zeta \in I\left(\Omega_{2}\right)$. Consider $B_{n}+\phi, B_{n} \varepsilon \Omega_{2}$ then $B_{n} \subset A_{n}^{\prime}+\phi, A_{n} \varepsilon \Omega_{1}$ since $\Omega_{2}$ is countably paracompact and $\Omega_{1}$ separates $\Omega_{2}$ therefore $\Omega_{2}$ is $\Omega_{1}$-countably paracompact and $\zeta\left(B_{n}\right)<\zeta\left(A_{n}^{\prime}\right)=\mu\left(A_{n}^{\prime}\right) \rightarrow 0$. Since $\mu \varepsilon I_{\sigma}\left(\Omega_{1}^{\prime}\right)$, then $\zeta \in I_{\sigma}\left(\Omega_{2}\right)$. Now extend $v_{1}, v_{2}$ to $\tau_{1}, \tau_{2} \varepsilon I_{R}\left(\Omega_{2}\right)$ then $\zeta<\tau_{1}\left(\Omega_{2}\right), \zeta<\tau_{2}\left(\Omega_{2}\right)$ by separation and so $\tau_{1}=\tau_{2}$ since $\Omega$ is mildly normal. Thus $\nu_{1}=\nu_{2}$ and so $\Omega_{1}$ is slightly normal.

REMARK 3.1. It is not difficult to give similar conditions (as in theorem 3.10) to obtain the other partial converse of theorem 3.9.

4. ON SPACES RELATED TO THE GENERAL WALLMAN SPACE.

We recall from section 2 that for an arbitrary lattice $\Omega$ of subsets of $X, I_{R}(\Omega)$ with the topology $T W(\Omega)$ of the closed set, is a compact $T_{1}$ space. Also, if $\Omega$ is disjunctive and separating then $X$ can be embedded in $I_{R}(\Omega)$. Moreover, if $\Omega$ is disjunctive, so is $W(\Omega)$, and $I_{R}(\Omega)$ with the topology $T(\Omega)$ is $T_{2}$ iff $\Omega$ is normal. In this section, we consider alternate topologies on $I_{R}(\Omega)$.

THEOREM 4.1. Consider $\delta=\tau W\left(\Omega^{\prime}\right)$ for a base of closed sets $W\left(L^{\prime}\right) ; L^{\prime} \varepsilon \Omega$ Then $I_{R}(\Omega)$ with the topology $\delta$ is $T_{2}$.

PROOF. If $\mu_{1} \neq \mu_{2}: \mu_{1}, \mu_{2} \varepsilon I_{R}(\Omega)$ then there exists $L_{1}, L_{2} \varepsilon \Omega ; L_{1} \cap L_{2}=\phi, \mu_{1}\left(L_{1}\right)=1$ and $\mu_{1}\left(\mathrm{~L}_{2}\right)=0, \mu_{2}\left(\mathrm{~L}_{2}\right)=1$ and $\mu_{2}\left(\mathrm{~L}_{1}\right)=0$. Therefore $\mu_{1} \varepsilon \mathrm{W}\left(\mathrm{L}_{1}\right), \mu_{2} W\left(\mathrm{~L}_{1}\right)$ and $\mu_{2} \varepsilon W\left(L_{2}\right), \mu_{1} \notin W\left(L_{2}\right) ; W\left(L_{1}\right)$ and $W\left(L_{2}\right)$ are open sets. Thus, $W\left(L_{1}\right) \cap W\left(L_{2}\right)=\phi$ and consequent $1 y, I_{R}(\Omega)$ with $\delta$ is $T_{2}$.

DEFINITION 4.1. $\Omega$ is said to be almost compact, if for all $\mu \varepsilon I_{R}\left(\Omega^{\prime}\right), S(\mu) \neq \phi$ THEOREM 4.2. The lattice $W\left(\Omega^{\prime}\right)$ is almost compact.

PROOF. Let $\lambda \varepsilon I_{R} W\left(\left(\Omega^{\prime}\right)^{\prime}\right)=I_{R}(W(\Omega))$ then $\lambda=\hat{\mu}, \mu \varepsilon I_{R}(\Omega)$ (section 2 ) and so $S(\hat{\mu})=n W(L)$ with $1=\hat{\mu}(W(L))=\mu(L)$. Thus, $\mu \varepsilon W(L), \mu \varepsilon S(\hat{\mu})$ on $W(\Omega)$ and so $I_{R}(\Omega)$ with $W\left(\Omega^{\prime}\right)$ is almost compact.

REMARK 4.1. We note (i) $S(\lambda)=S(\hat{\mu})=\{\mu\}$. (ii) If $\Omega$ is disjunctive, it is then clear that for any $L \in \Omega, L=\cap L_{\alpha}^{\prime}, L \subset L_{\alpha}^{\prime} ; L_{\alpha} \varepsilon \Omega$.

THEOREM 4.3. The sets of $W(\Omega)$ are clopen in the $\delta$ topology.

PROOF. Since $W(L)$ is disjunctive then by remark 4.2 (ii) for any $L \varepsilon \Omega$, $W(L)=r_{1} W\left(L_{\alpha}^{\prime}\right), W(L) \subset W^{\prime}\left(L_{\alpha}\right), L_{\alpha} \varepsilon \Omega$ is closed in the $\delta$-topology. But, $W(L)$ is also open in $\delta$-topology since $W(L)=\left(W^{\prime}\left(L^{\prime}\right)^{\prime}\right)$. Thus, the sets of $W(\Omega)$ are closed in the $\delta$-topology.

THEOREM 4.4. $I_{R}(\Omega)$ with the $\delta$-topology is compact iff $\Omega$ is an algebra.

PROOF. (1) Suppose $I_{R}(\Omega)$ with $\delta$ is compact. Thus, ${ }^{W}\left(\Omega^{\prime}\right)$ is a compact lattice and so is $W\left(\Omega^{\prime}\right)$. Let $\zeta \in I\left(W\left(\Omega^{\prime}\right)\right)$. Then $S(\zeta) \neq \phi$ on $W\left(\Omega^{\prime}\right), \zeta=\hat{\lambda} ; \lambda \varepsilon I(\Omega)$. 
Let $\mu \varepsilon S(\zeta), \mu \varepsilon I_{R}(\Omega)$ then $1=\zeta\left(W\left(L^{\prime}\right)\right)$ iff $\hat{\lambda}\left(W\left(L^{\prime}\right)\right)=1$ iff $\lambda\left(L^{\prime}\right)=1$. Now $\lambda\left(L^{\prime}\right)=1$. Then $\zeta\left(W\left(L^{\prime}\right)\right)=1$ then $\mu \varepsilon W\left(L^{\prime}\right)$ then $\mu\left(L^{\prime}\right)=1$. Thus, $\lambda<\mu\left(\Omega^{\prime}\right)$ and hence $\mu<\lambda(L)$. But $\mu \in I_{R}(L)$ then $\mu=\lambda$. Thus $\hat{\mu}=\hat{\lambda}$ and hence $I(W(\Omega)$ ) $=I\left(W\left(\Omega^{\prime}\right)\right)=I_{R}(W(\Omega))$. Thus, $\Omega=\Omega^{\prime}[2]$. (11) The converse is clear.

THEOREM 4.5. $\delta=\tau^{\mathrm{W}}(\Omega)$ iff $\Omega$ is an algebra.

PROOF. (i) Since the sets $W(\Omega)$ are clopen by Theorem 4.3 then $W(\Omega) \varepsilon \delta$ and so $\tau W(\Omega) \varepsilon \delta$. Now, if $\delta=\tau W(\Omega)$ then since $\mathrm{TW}(\Omega)$ is compact so $1 \mathrm{~W} \delta$ and $80 \Omega$ is an algebra by theorem 4.4. (11) The converse is clear.

In the next theorem, we give another equivalent condition for $\Omega$ to be an algebra.

THEOREM 4.6. $\Omega$ is an algebra iff $W\left(\Omega^{\prime}\right)$ is a disjunctive lattice in $I_{R}(\Omega)$.

PRoOF. (i) Suppose $\Omega$ is an algebra, then $I_{R}(\Omega)=I(\Omega)$. Let $\mu \in I_{R}(\Omega)$;

$\mu \notin W\left(L^{\prime}\right), L \varepsilon \Omega$. Then $\mu\left(L^{\prime}\right)=0,1=\mu(L)=\mu\left(\left(L^{\prime}\right)^{\prime}\right) . L^{\prime} \varepsilon \Omega$ and $W\left(L^{\prime}\right) \cap W\left(\left(L^{\prime}\right)\right)^{\prime}$

$=\phi$. Thus, $W\left(\Omega^{\prime}\right)$ is disjunctive. (1i) Suppose $W\left(\Omega^{\prime}\right)$ is disjunctive. Let

$\mu \varepsilon I(\Omega)$ then there exists a $\nu \varepsilon I_{R}(\Omega) ; \mu<\psi(\Omega)$. For $\mu \neq \nu$, there exists

$\mathrm{L} \varepsilon \Omega, \mu(L)=0, U(L)=1$ then $v \notin W\left(L^{\prime}\right)$. Hence, by disjunctiveness

$\nu \varepsilon W(\hat{L})^{\prime}, L \in \Omega$ and $\phi=W(\hat{L})^{\prime} \cap W(L)^{\prime}=W\left(\hat{L} \prime^{\prime} \cap L^{\prime}\right)=W\left((\hat{L} \cup L)^{\prime}\right)$. Hence, $\hat{\mathrm{L}} \cup \mathrm{L}_{\mathrm{l}}=\mathrm{X}, \mathrm{L}^{\prime} \hat{\mathrm{L}}^{\prime}=\phi$ and so $\mathrm{L}^{\prime} \subset \hat{\mathrm{L}}$, but since $\mu(\hat{\mathrm{L}})=1$ and $\nu \varepsilon W(\hat{\mathrm{L}})^{\prime}, \nu(\hat{\mathrm{L}})=0$ which is a contradiction, since $\mu<\nu$. Thus, $I_{R}(\Omega)=I(\Omega)$, and so $\Omega$ is an algebra.

\section{ON NON-DISJUNCTIVE LATTICES.}

We next consider the case where $\Omega$ is not necessarily disjunctive. We begin, by introducing the notion of an s-convergent measure and some related results and then proceed to the construction of an analogue of the Wallman space.

DEFINITION 5.1. $\mu \in I(\Omega)$ is said to be $\Omega$-convergent if there exists an $x \in \mathrm{X}$ such that $\mu_{x}<\mu(\Omega)$.

THEOREM 5.1. $\mu$ is $\Omega$-convergent iff $S(\mu) \neq \phi$ on $\Omega^{\prime}$, for all $\mu \varepsilon I(\Omega)$.

PROOF. (1) Suppose $\mu$ is $\Omega$-convergent. Then there exists $x \in X ; \mu_{x}<\mu(\Omega)$ and so $\mu<\mu_{x}\left(\Omega^{\prime}\right)$. Moreover $x \in S\left(\mu_{x}\right) \subset S(\mu)$ on $\Omega^{\prime}$. Thus, $S(\mu) \neq \phi$ on $\Omega^{\prime}$.

Suppose $S(\mu) \neq \phi$ on $\Omega^{\prime}$ for $\mu \varepsilon I(\Omega)$. Let $x \in S(\mu)$ on $\Omega^{\prime}$. Then, $\mu<\mu_{x}\left(\Omega^{\prime}\right)$ and so

$\mu_{x}<\mu(\Omega)$. Thus $\mu$ is $\Omega$-convergent.

THEOREM 5.2. Suppose $\mu_{1}<\mu_{2}(\Omega)$, for all $\mu_{1}, \mu_{2} \varepsilon I(\Omega)$. Then

a) If $\mu_{1}$ is $\Omega$-convergent so is $\Omega_{2}$.

b) If $\Omega^{\prime}$ is regular and $\mu_{2}$ is $\Omega$-convergent then $\mu_{1}$ is $\Omega$-convergent.

PROOF. a) Suppose $\mu_{1}$ is $\Omega$-convergent, then $\mu_{x}<\mu_{1}(\Omega)$ for some $x$, but

$\mu_{1}<\mu_{2}(\Omega)$ then $\mu_{x}<\mu_{2}(\Omega)$ and so $\mu_{2}$ is $\Omega$-convergent. b) Suppose $\mu_{2}$ is

s-convergent, then $\mu_{x}<\mu_{2}(\Omega)$ for some $x$ and so $\mu_{2}<\mu_{x}\left(\Omega^{\prime}\right)$ and $x \in S\left(\mu_{2}\right)$ on $\Omega^{\prime}$ but $\mu_{1}<\mu_{2}(\Omega)$ then $\mu_{2}<\mu_{1}\left(\Omega^{\prime}\right)$ and since $\Omega^{\prime}$ is regular $\times \in S\left(\mu_{2}\right)=S\left(\mu_{1}\right)$ on $\Omega^{\prime}$. Thus, $S\left(\mu_{1}\right) \neq \phi$ on $\Omega^{\prime}$ and so $\mu_{1}$ is $\Omega$-convergent.

THEOREM 5.3. Suppose $\Omega^{\prime}$ is $\mathrm{T}_{2}$ and $\mu$ is $\Omega$-convergent where $\mu \in I(\Omega)$. Then, there exists a unique $x \in X: \mu_{x}<\mu\left(\Omega^{\prime}\right)$.

PROOF. If $\Omega^{\prime}$ is $T_{2}$ and if $x \neq y$ then there exists $L_{1}, L_{2} \varepsilon \Omega ; x \in L_{1}$, $y \varepsilon L_{2} ; L_{1} \cap L_{2}=\phi$ and $\mu_{x}\left(L_{1}\right)=1, \mu_{x}\left(L_{2}\right)=1$. Now, if $\mu_{x}<\mu(\Omega)$ and $\mu_{y}<\mu(\Omega)$ then $\mu\left(L_{1}\right)=\mu\left(L_{2}\right)=1$ but $L_{1} \cap L_{2}=\phi$ which is a contradiction and so the desired result is true. 
DEFINITION 5.2. $\Omega$ is said to be weakly compact te for all $\mu \varepsilon I_{R}(\Omega), \mu$ is s-convergent.

DEFINITION 5.3. $\Omega$ is said to be almost compact if for any $\mu \varepsilon I_{R}\left(\Omega^{\prime}\right), \quad S(\mu) \neq \phi$ on $\Omega$.

THEOREM 5.4. $\Omega$ is weakly compact iff $\Omega^{\prime}$ is almost compact.

PROOF. (i) Let $\mu \varepsilon I_{R}(\Omega)$ then since $\Omega$ is weakly compact, there exists an $x \varepsilon x$ such that $\mu_{x}<\mu(\Omega)$ and $S(\mu) \neq \phi$ on $\Omega^{\prime}$. Thus, $\Omega^{\prime}$ is almost compact. (ii) Let $\mu \varepsilon \mathrm{I}_{\mathrm{R}}(\Omega)$ then $\mathrm{S}(\mu) \neq \phi$ on $\Omega^{\prime}$ since $\Omega$ is almost compact. Let $\mathrm{x} \varepsilon \mathrm{S}(\mu)$ on $\Omega^{\prime}$ then $\mu<\mu_{x}\left(\Omega^{\prime}\right), \mu_{x}<\mu(\Omega)$ and so $\Omega$ is weakly compact.

Now, note that a topological space $X$ is absolutely closed (generalized absolutely closed) iff the lattice $\theta$ of open sets is $T_{2}\left(T_{0}\right)$ and weakly compact. Next, let $\Omega$ be a lattice of subsets of $X$ and define $U(\Omega)=\left\{U L{ }_{\alpha} ; L_{\alpha} \varepsilon \Omega\right\}$.

THEOREM 5.5. a) If $\Omega_{1} \subset \Omega_{2}$ and $\Omega_{2}$ is weakly compact, then $\Omega_{1}$ is weakly compact. b) Suppose $\Omega_{1} \subset \Omega_{2} \subset U\left(\Omega_{1}\right)$ and $\Omega_{2}$ semi-separates $\Omega_{2}$. Then, if $\Omega_{1}$ is weakly compact, $\Omega_{2}$ is weakly compact.

PROOF. a) Extend $\mu \in I_{R}\left(\Omega_{1}\right)$ to $\nu \in I_{R}\left(\Omega_{2}\right)$. Since $\Omega_{2}$ is weak1y compact, there exists an $x ; \mu_{x}<v\left(\Omega_{2}\right)$. $\mu_{x}<\mu\left(\Omega_{1}\right)$ and so $\Omega_{1}$ is weakly compact. b) Let $v \varepsilon I_{R}\left(\Omega_{2}\right)$. Then since $\Omega_{1} s . s \Omega_{2}, \mu \varepsilon I_{R}\left(\Omega_{1}\right)$ where $\mu$ is the restriction of $\nu$ to $A\left(L_{1}\right)$. Thus, there exists an $x \in X$ such that $\mu_{x}<\mu\left(\Omega_{1}\right)$. Now, suppose $L_{2} \varepsilon \Omega_{2}$ and $\mu_{x}\left(L_{2}\right)=1$ then $x \varepsilon X$ $\mathrm{X} \varepsilon \mathrm{L}_{2}$, but $\mathrm{L}_{2}=U \mathrm{~L}_{1 \alpha}, \mathrm{L}_{1 \alpha} \varepsilon \Omega$; then $\mathrm{x}$ is on some $\mathrm{L}_{1 \alpha}$ and so $\mu_{\mathrm{x}}\left(\mathrm{L}_{1 \alpha}\right)=1$ for some $\mathrm{L}_{1 \alpha}$ and moreover $\mu\left(L_{1 \alpha}\right)=1$ since $\mu_{x}<\mu\left(\Omega_{1}\right)$, but $L_{1 \alpha} \subset L_{2}$ then $\nu\left(L_{2}\right)=1$ and so

$\mu_{x}<v\left(\Omega_{2}\right)$. Thus, $\Omega_{2}$ is weakly compact.

REMARK 5.1. Let $X$ be a topological space and $\theta$ the collection of open sets. Then, by Theorem 5.4, $\theta$ is weakly compact iff $F=\theta^{\prime}$ is almost compact.

Now consider $X$. Suppose $\Omega$ is non-disjunctive and define $\hat{I}=\left\{\mu_{x}: x \in X\right\} U$ $\left\{\mu \varepsilon I_{R}(\Omega): \mu\right.$ is not $\Omega$-convergent $\}$ and $\hat{W}(A)=\{\mu \varepsilon \hat{I} ; \mu(A)=1, A \varepsilon A(\Omega)\}$. We also assume $\Omega$ is $T_{0}$, so $x, y \in X$ and $x \neq y$ implies $\mu_{x} \neq \mu_{y}$.

THEOREM 5.6. For $A, B \in A(\Omega)$, we have: a) $A=B$ iff $\hat{W}(A)=\hat{W}(B), \quad b) \hat{W}(A \cup B)=$ $\hat{W}(A) \cup \hat{W}(B)$, c) $\hat{W}(A \cap B)=\hat{W}(A) \cap \hat{W}(B)$, d) $\hat{W}\left(A^{\prime}\right)=(\hat{W}(A))^{\prime}$, e) $\hat{W}(A(\Omega))=A(\hat{W}(\Omega))$. PROOF. a) (i) If $A=B$, then, clearly $\hat{W}(A)=\hat{W}(B)$. (1i) If $A \neq B$, then say $A \cap B^{\prime} \neq \phi$, let $x \in A \cap B^{\prime}$ then $\mu_{x}\left(A \cap B^{\prime}\right)=1 ; \mu_{x} \varepsilon \hat{I}$ and so $\mu_{x}(A)=1, \mu_{x}(B)=0$ which Implies that $\mu \in \hat{W}(A), \mu \notin \hat{W}(B)$ and $\hat{W}(A) \neq \hat{W}(B)$, b), c), d) and e) are not difficult to show and are omitted.

Now consider $\mu \varepsilon I(\Omega)$ and define $\hat{\mu} \varepsilon I(\hat{W}(\Omega))$ to be $\hat{\mu}(\hat{W}(A))=\mu(A), A \varepsilon A(\Omega)$. Then, one can easily note that $\mu \rightarrow \hat{\mu}$ is $1-1$ and onto from $I(\Omega)$ to $I(\hat{W}(\Omega))$, and moreover $\mu \varepsilon I_{R}(\Omega)$ iff $\hat{\mu} \varepsilon I_{R}(\hat{W}(\Omega))$.

THEOREM 5.7. $\hat{W}(\Omega)$ is weakly compact and $T_{0}$.

PROOF. a)Let $\hat{\mu} \varepsilon I_{R}(\hat{W}(\Omega))$ then $\mu \varepsilon I_{R}(\Omega)$. If $\mu$ is $\Omega$-convergent then $\mu_{x}<\mu(\Omega)$ implies that $\hat{\mu}_{x}<\hat{\mu}(\hat{W}(\Omega))$. Note that for $A \in A(\Omega)$, $\mu_{x}(A)=\hat{\mu}_{x}(\hat{W}(A))=\left\{\begin{array}{l}1 \text { if } x \in A \\ 0 \text { if } x \notin A\end{array}\right.$ and $\mu_{x} \varepsilon \hat{W}(A)$ iff $\mu_{x}(A)=1$. Thus, $\hat{\mu}_{x}$ is the measure concentrated at $\mu_{x}$ and so $\hat{\mu}$ is $\hat{W}(\Omega)$-convergent. If $\mu$ is not $\Omega$-convergent then $S(\hat{\mu})=n \hat{W}(L)$ with $\hat{\mu}(\hat{W}(L))=1=\mu(L)$, hence $\mu \varepsilon \hat{W}(L), \mu \varepsilon I, \mu \varepsilon S(\hat{\mu})$ and $\hat{\mu}$ is the measure concentrated at $\mu$, and consequent $1 \mathrm{y} \hat{W}(\Omega)$ is weakly compact. b) Let $\mu_{1}$, 
$\mu_{2} \varepsilon \hat{I}, \mu_{1} \neq \mu_{2}$ then there exists an $L \varepsilon \Omega$ such that say, $\mu_{1}(L)=1, \mu_{2}(L)=0$. Therefore $\mu_{1} \varepsilon \hat{w}(L), \mu_{2} \varepsilon \hat{w}\left(L^{\prime}\right)$ and so $\hat{w}(L)$ is $T_{0}$.

THEOREM 5.8. If $\Omega=U(\Omega)$ then $\hat{W}(\Omega)$ separates $U(\hat{W}(\Omega))$.

PRoof. Suppose (i) $\left(\cup_{\alpha} \hat{W}\left(L_{\alpha}\right) \cap\left(\underset{\beta}{U}\left(L_{\beta}^{\prime}\right)\right)=\phi\right.$. Let $A=\left(\bigcup_{\alpha} L_{\alpha}\right) \in \Omega, B=\left(\cup_{\beta} L_{\beta}\right) \varepsilon \Omega$.

Since $\Omega=U(\Omega)$ then $\left(\underset{\alpha}{W}\left(L_{\alpha}\right)\right) \subset \hat{W}(A)$ and $\left(U_{\beta} \hat{W}\left(L_{\beta}\right) \subset W(B)\right.$. If $A \cap B \neq \phi$ then $L_{\alpha} \cap L_{\beta} \neq \phi$

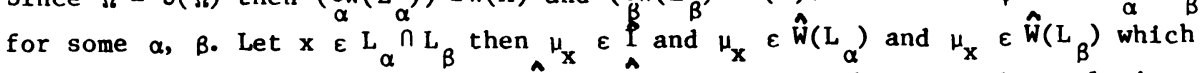
contradicts (i). Thus, $A \cap B=\phi, \hat{w}(A) \cap \hat{W}(B)=\phi$, and the desired result is now clear.

Now, we note that if $\Omega=U(\Omega)$ then $\hat{I}$ with $\theta=\hat{W}(\Omega)$ is generalized absolutely closed and is absolutely closed if $\Omega^{\prime}$ is $T_{2}$. Thus, if we consider $X$ and let $\Omega=U(\Omega)=\theta_{x}$ and $T_{2}$, then $\hat{I}, \theta$ is an absolute closure of $X$ since one can easily observe that $\bar{x}=\hat{w}(X)$.

REMARK. An analogous construction can now be done for $\hat{I}^{\sigma}, \hat{w}^{\sigma}$. Where $\hat{I}^{\sigma}=\left\{\mu_{X}: x \in X\right\} \cup\left\{\mu \varepsilon I_{R}^{\sigma}(\Omega): \mu\right.$ is not $\Omega$-convergent $\}$ and $\hat{W}^{\sigma}(A)=\left\{\mu \varepsilon I^{\sigma}\right.$, $\mu(A)=1, A \in A(\Omega)$ and one can show that $\hat{I}^{\sigma}(\Omega)$ is weakly replete. $\Omega$ is weakly replete if for any $\mu \in \mathrm{I}_{\mathrm{R}}^{\sigma}(\Omega)$, $\mu$ is $\Omega$-convergent. We note that the constructions here generalize the work of Lui [6].

REFERENCES

1. NOEBELING, G., Gründlagen der analytischen topologie, Springer-Verlag, Berlin 1954.

2. FROLIK, Z., Prime Filters with the C.I.P., Comm. Math. Univ. Carolinae, 13 (1972), 533-575.

3. BACHMAN, G. and STRATIGOS, P., Criteria for $\sigma$ smoothness, , T-smoothness, and tightness of Lattice Regular Measures, with Applications, Con. J. Math. 33(1981), 1498-1525.

4. GRASSI, P., On subspaces of replete and measure replete spaces. Con. Math. Bu11. 27(1), (1984), 58-64.

5. SZETO, M., Measure Repleteness and Mapping Preservations, J. Indian Math. Soc. 43(1979), 35-52.

6. LIU, C.T., Absolutely Closed Spaces, Trans. Amer. Math. Soc. 130 (1968), 86-104.

7. BoURbaKI, N., Elements of Mathematics, General Topology, Part I, AddisonWesley, Reading, Mass. 131. 


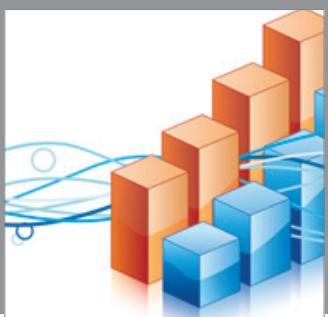

Advances in

Operations Research

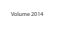

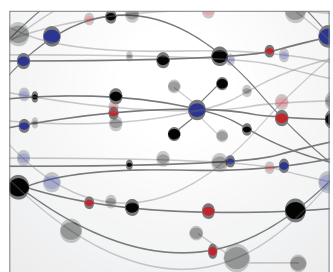

\section{The Scientific} World Journal
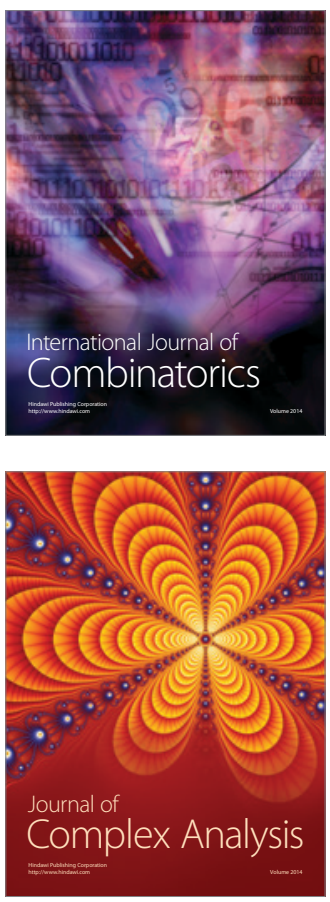

International Journal of

Mathematics and

Mathematical

Sciences
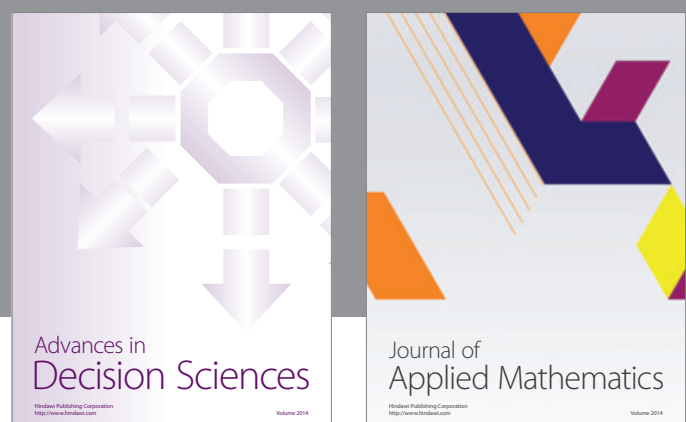

Journal of

Applied Mathematics
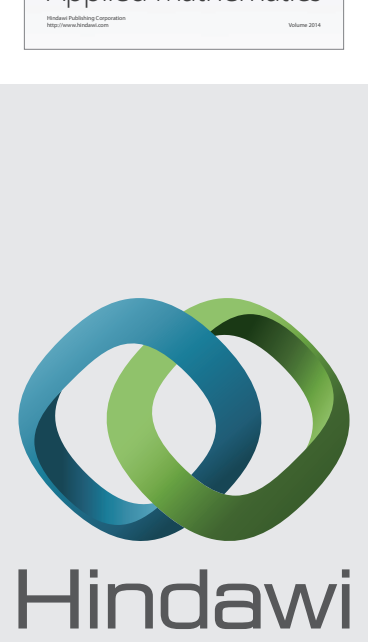

Submit your manuscripts at http://www.hindawi.com
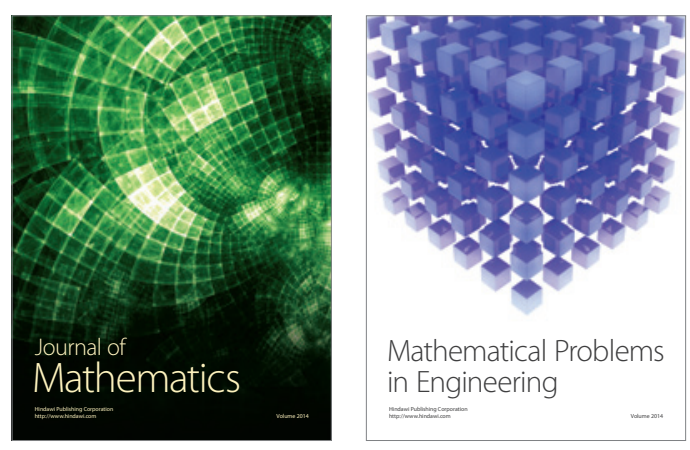

Mathematical Problems in Engineering
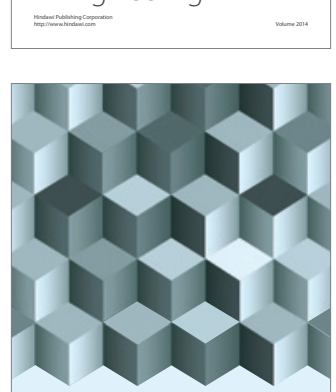

Journal of

Function Spaces
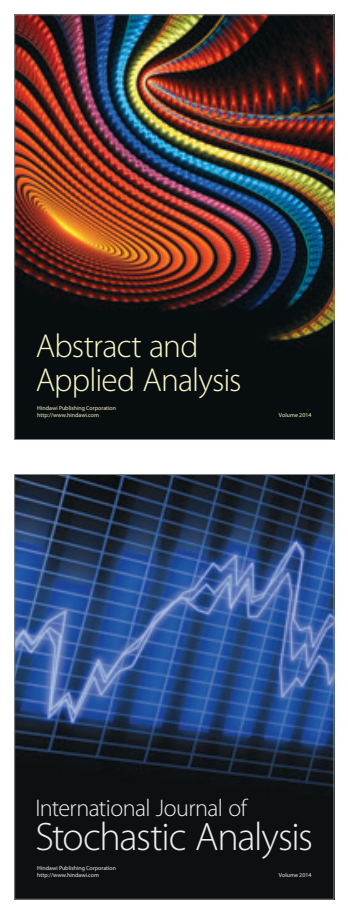

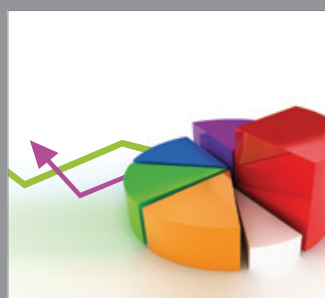

ournal of

Probability and Statistics

Promensencen
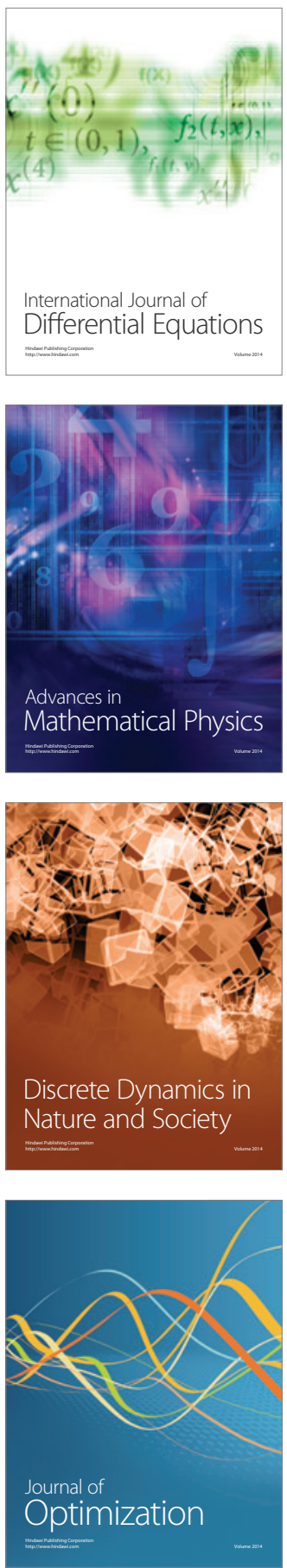